Malaysian Journal of Fundamental and Applied Sciences

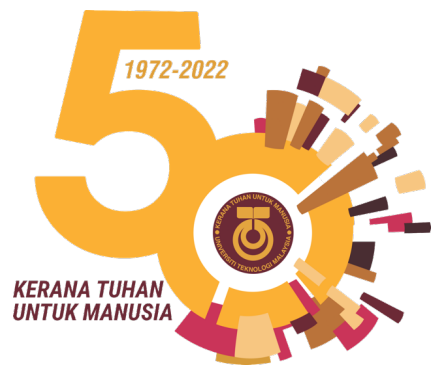

\title{
In-silico Studies Reveal Potential Epitope based Vaccine against M.leprae Phosphoglycerate Mutase Protein
}

\author{
Renata Triwijaya, Winda Hasuki, Gabriele Kresnia, Jacqulin Natasya, Putri \\ Satya, Arli Aditya Parikesit*
}

Bioinformatics Department, School of Life Science, Indonesia International Institute for Life Sciences, Jakarta 13210, Indonesia

Abstract Leprosy is an infectious disease caused by Mycobacterium leprae that mainly affects the skin, peripheral nerve, mucosa of the upper respiratory tract, and eyes. There is no vaccine designed specifically to prevent leprosy. The most common vaccine strategy is Bacille Calmette-Guérin (BCG), however its efficacy is highly variable between studies. Current study utilized a computational method to predict antigenic epitopes from Mycobacterium leprae for peptide vaccine development. Molecular docking of top predicted peptides from 6 antigenic B-cell and 3 CTL epitopes were analyzed. These predicted antigenic epitopes might potentially be target peptides for future leprosy vaccines.

Keywords: computational, leprosy, phosphoglycerate mutase, vaccine, epitopes, CTL, MHC.

\section{Introduction}

Leprosy is an infectious disease caused by Mycobacterium leprae that mainly affects the skin, peripheral nerve, mucosa of the upper respiratory tract, and eyes. In Indonesia, leprosy was a serious issue. There were a total of 126,221 incidents found in 1985. The prevalence was decreasing by $86 \%$ after 15 years, and the Indonesian government declared the elimination of leprosy was achieved in 2000 [1]. However, in 2018, 14,397 new cases were detected. By 2020, Indonesia was still ranked 3rd with leprosy [2].

There is no vaccine designed specifically to prevent leprosy. The most common vaccine strategy is Bacille Calmette-Guérin (BCG), which has been used to prevent both leprosy and tuberculosis [3]. The BCG vaccine is the live attenuated vaccine form of Mycobacterium bovis. This vaccine was designed as a tuberculosis vaccine but was speculated to give protection against leprosy due to the similar antigenic makeup of the causing agents [3][4].

*For correspondence: arli.parikesit@i3l.ac.id

Received: 22 June 2021

Accepted: 25 Dec 2021

(C) Copyright Triwijaya et al. This article is distributed under the terms of the Creative Commons Attribution License, which permits unrestricted use and redistribution provided that the original author and source are credited.
According to Duthie, Gillis, and Reed [5], the effect of the BCG vaccine on leprosy is highly variable between studies; the efficacy was shown to be $26-41 \%$ in experimental studies and $61 \%$ in observational studies. Besides that, leprosy is still endemic in countries where BCG vaccination is common.

There are many methods to identify novel vaccine and drug targets. One example is a previous study by Jaiswal et al. [6], who screened M.leprae's homologous conserved proteome for secreted, membrane and putative surface exposed proteins, in which the identified genes were analyzed further as potential vaccine candidates and docked.

This study screens for potential epitopes of a specific gene of M.leprae. The study determines potential B-cell and T-cell epitopes of M.leprae's GPM1 that have strong affinity to major histocompatibility complexes (MHC). The specific gene is chosen because it encodes phosphoglycerate mutase (PGM), an important protein in the glycolysis metabolic pathway [7]. The study aims to find the potential peptide vaccine that inhibits M.leprae's phosphoglycerate mutase.

Prediction of B-cell and cytotoxic $\mathrm{T}$ lymphocytes (CTL) epitopes are essential processes in vaccine 
development. By employing the in-silico method, current research will analyze potential B-cell and T-cell epitopes to obtain effective vaccine candidates. Moreover, the selection of peptides from the pathogen proteome that has strong affinity to major histocompatibility complexes (MHC) is also crucial. Numerous bioinformatics tools are utilized to model and analyze the peptide-MHC complexes for effective vaccine development

\section{Materials and Methods}

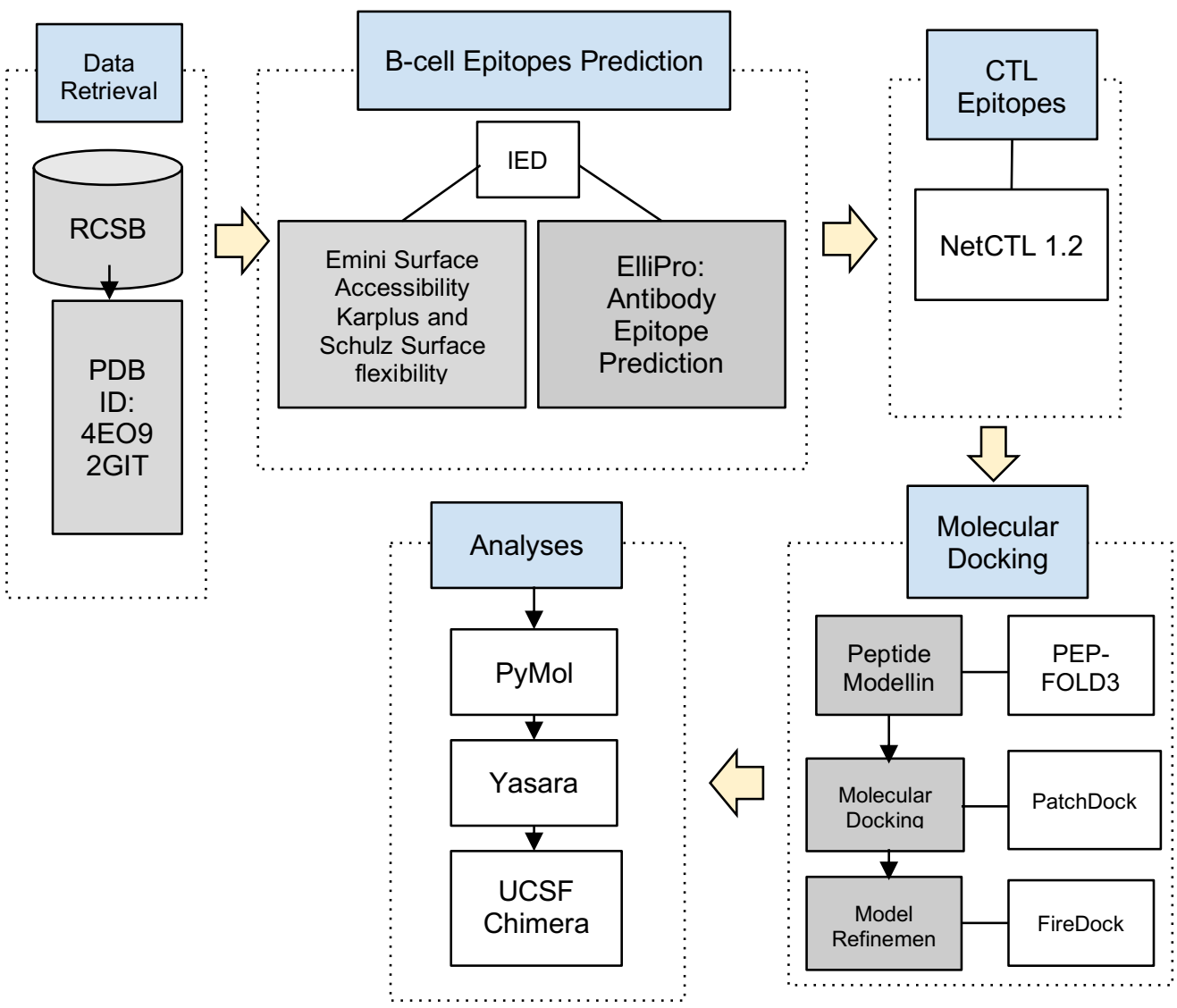

Figure 1. Overall Workflow

\section{Data Retrieval}

The crystal structures were obtained from Protein Data Bank. M. leprae phosphoglycerate mutase (PGM) GPM1 with PDB ID: 4EO9 and MHC Class I HLA-A2 with PDB ID: 2GIT. The amino acid sequence of PGM was also extracted from the crystal structure.

\section{Prediction of linear and conformational B-cell epitopes}

The the immune epitope database and analysis resource (IEDB) (http://www.iedb.org/) was utilized to predict surface accessibility based on Emini's method [8], predict flexibility based on Karplus and Schulz's method [9], predict hydrophilicity based on Parker's method [10], and predict antigenicity based on Kolaskar and Tongaonkar [11]. The conformational of B-cell epitopes was predicted with ElliPro (http://tools.iedb.org/ellipro/) [12]. The analysis uses three algorithms, which are approximation of protein shape, Protrusion Index (PI) of residues, and PI-based adjacent residues clustering [5].

\section{Prediction of cytotoxic T-lymphocytes (CTL) epitopes}

The NetCTL 1.2 server [13] was employed to predict CTL epitopes. To submit to the server, the sequence in Fasta format was inputted to the server, then peptide lengths and human leukocyte antigen (HLA) were chosen. 
Molecular docking and analysis of peptide-MHC complex

Predicted antigenic CTL epitopes were selected to be 3D-modelled with PEP-FOLD3 server [13-15]. The higher scoring peptides were chosen for molecular docking with MHC I HLA-A2 by utilizing the PatchDock server with the complex type option antibody-antigen [16-17]. The model complexes were then refined with FireDock [18-20]. Firedock also predicted the docking scores. Molecular visualization was performed with PyMOL [21], Yasara [22], UCSF Chimera 1.15 [23].

\section{Results and Discussion}

The research identifies linear and conformational B-cell and T-cell epitopes to design peptide vaccines against leprosy. Computational analyses were carried out with molecular docking software to predict the conformational interactions between antigenic epitopes and MHC molecules as an effort to identify potential novel vaccines. In-silico methodologies to design vaccines have already been proven to be promising and vital as it helps reduce the load of vaccine candidates screening of in-vitro experiments [24].

The physicochemical of residues plays a major role in antigenic epitopes [11]. Kolaskar and Tongaonkar's antigenicity prediction method was utilized. It predicts antigenic determinants based on the psychochemical characteristics of residues and their occurrence frequencies and is proven to have $75 \%$ accuracy [11].

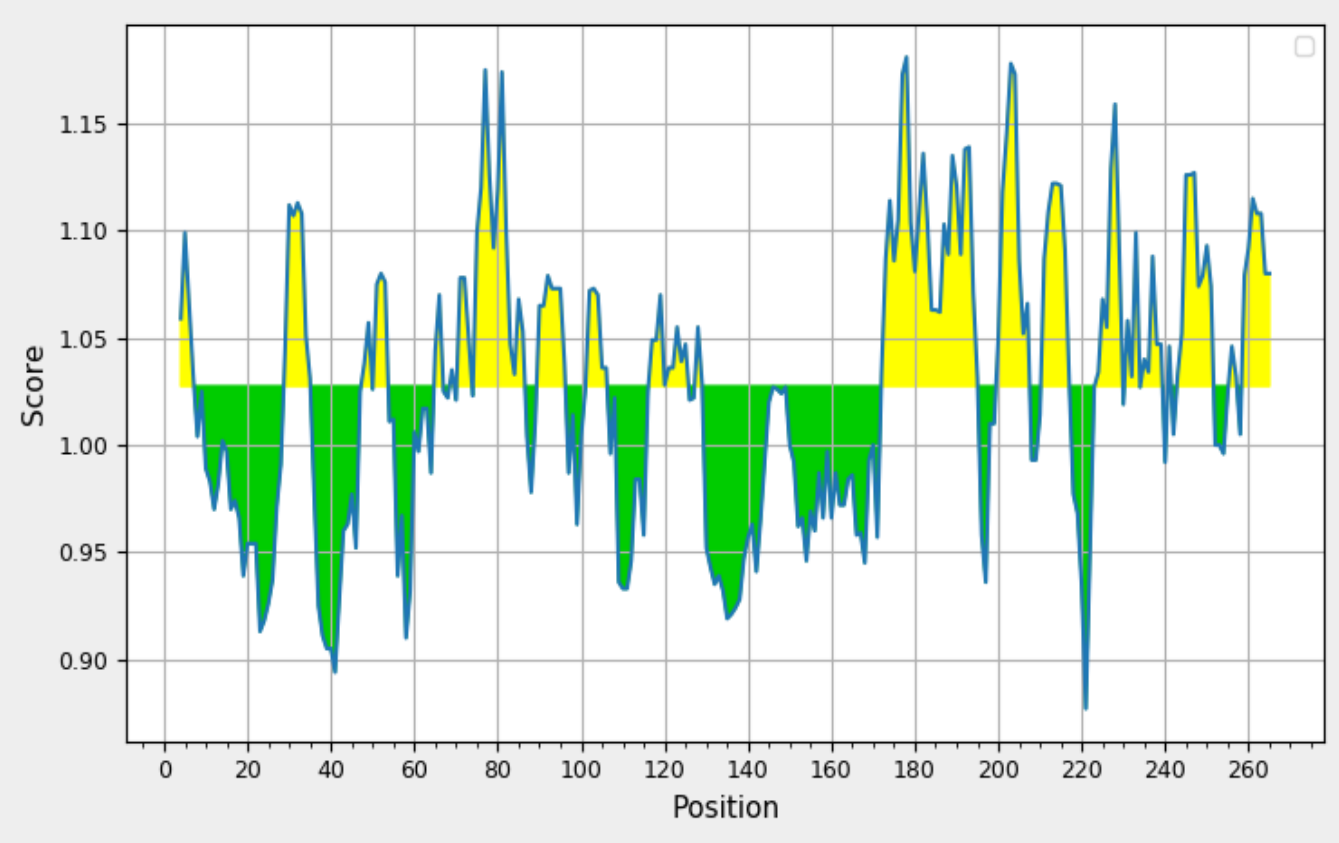

Figure 2. Antigenicity of M.leprae PGM with Kolaskar and Tongaonkar's scale. Residual score is represented along the $\mathrm{X}$-axis while sequence position is represented along $\mathrm{Y}$-axis.

A total of 6 antigenic epitopes have been predicted to occur within the range 8-42 amino acids from the complete sequence of M.leprae PGM (Table 1). By Kolaskar and Tongaonkar's calculation, there are 170 out of 265 residues exhibiting more than 1.000 residual score. This is shown graphically in figure 2 An observation also reveals that some of the predicted residues in B-cell epitopes are also found in CTL epitope prediction (Table 1 in bold). Thereby, the common residues at these positions are significant as potential peptide vaccine candidates. 
Table 1. Predicted antigenic B-cell epitopes of M.leprae PGM and common residues (in bold) found in B-cell and Cytotoxic T lymphocytes.

\begin{tabular}{ccccc}
\hline Start & End & Peptide & Number of Residues & Starfish \\
\hline 100 & 141 & GLDKAVTKARYGEERFMAWRRSYDTPPPPIEKGSEFSQDADP & 42 & 0.74 \\
\hline 75 & 85 & DTADWLWIPVR & 11 & 0.725 \\
\hline 218 & 229 & DLDADLRPVVPG & 12 & 0.717 \\
\hline 171 & 179 & VPDLRTGRT & 9 & 0.661 \\
\hline 144 & 152 & TDIGGGPLT & 9 & 0.655 \\
\hline 49 & 56 & AEHNLLPD & 8 & 0.638 \\
\hline
\end{tabular}

\section{Surface Accessibility of PGM}

Emini surface accessibility calculation is based on a product's surface where a hexapeptide with surface probability more than 1.0 indicates high probability that it will be on the surface [8]. Figure 3 is the graphical representation of the predicted peptides of PGM based on sequence position along the $X$-axis and surface accessibility prediction score along the $\mathrm{Y}$-axis. It has been observed that the maximum surface probability score is 4.489 and held by hexapeptide sequence RRSYDT within the range of 140 to 145 , while the minimum surface score is 0.095 with hexapeptide sequence CLADVV within the range of $175-180$ residues.

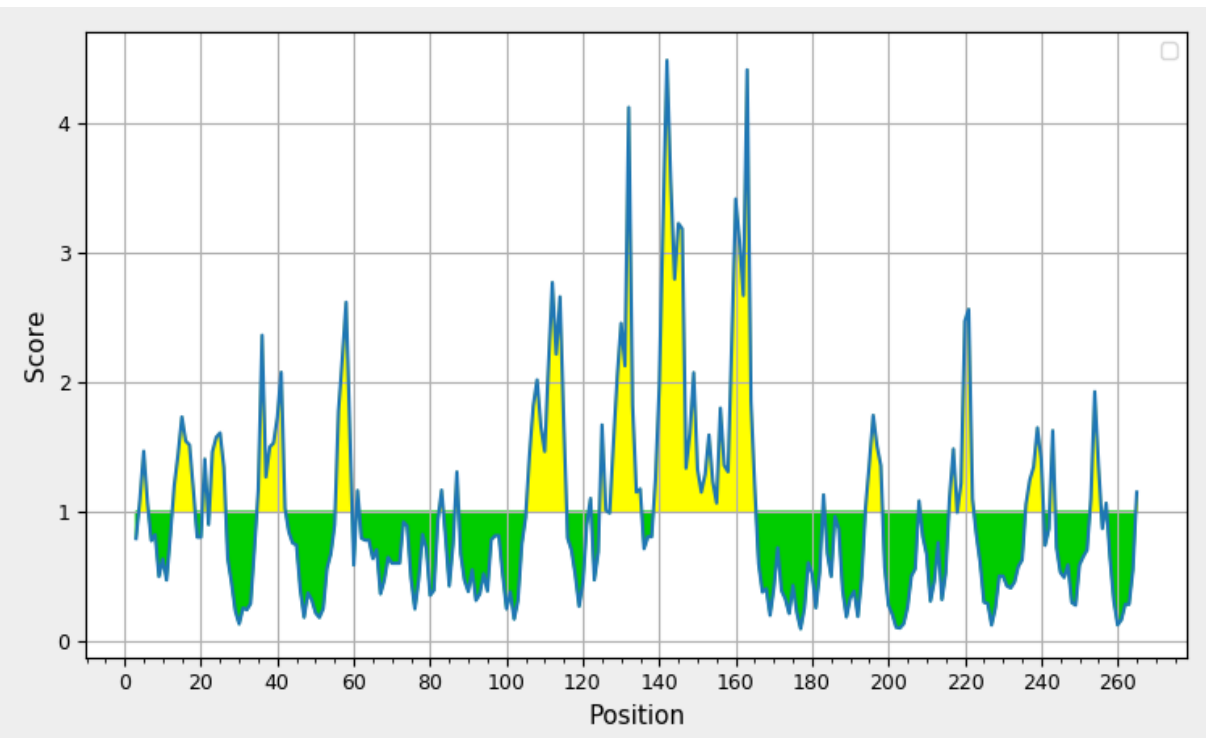

Figure 3. Emini Surface Accessibility Calculation for M.leprae PGM. Sequence position is along the Xaxis, while surface access probability is along the $\mathrm{Y}$-axis.

\section{Surface flexibility of PGM}

Karplus and Schulz' method calculates flexibility based on protein structure's atomic vibrational motions which are constructed through known temperature of B factors [9]. Lower B-factor values indicate that the model structure is well organized, while higher B-factor values indicate that the structure is unorganized and unordered [24]. The surface flexibility prediction was graphically represented in figure 4. The predicted maximum flexibility score is 1.108 at the range $15-21$ with the heptapeptide sequence QTQGPGS, while the minimum flexibility score is 0.891 at the range 99-105 with DWLWIPV heptapeptide sequence. 


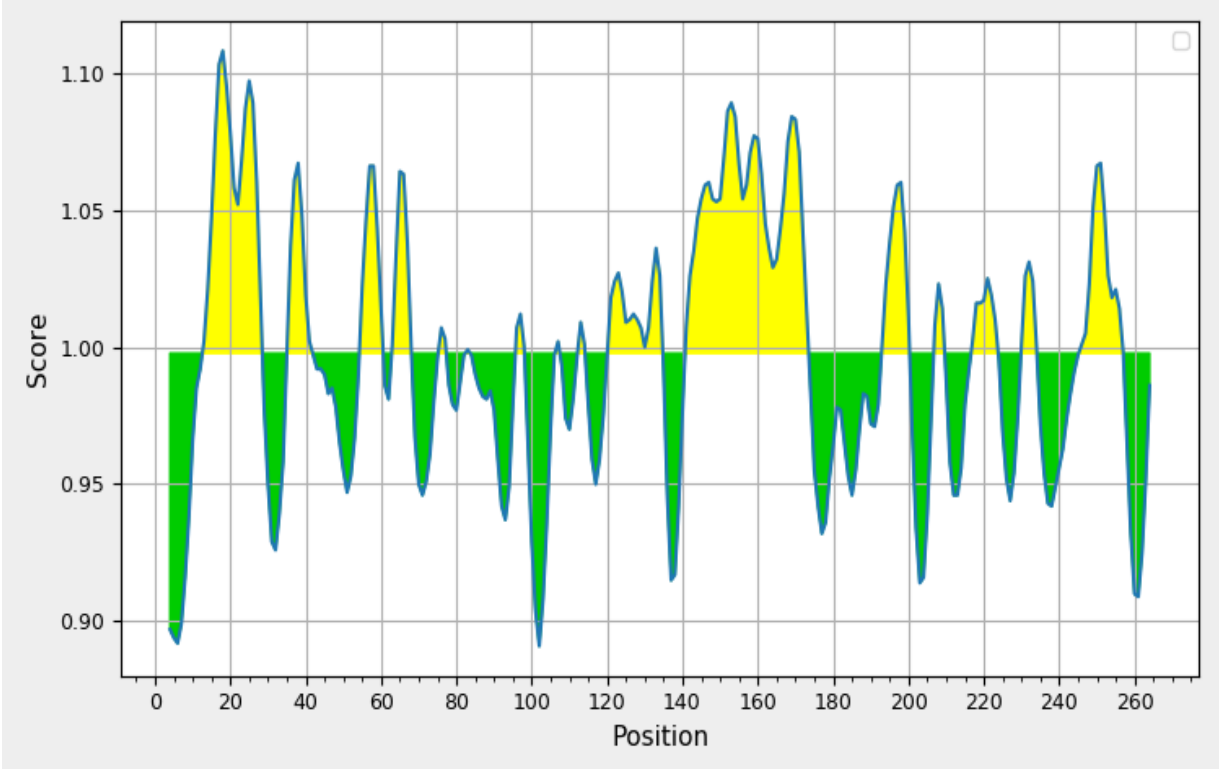

Figure 4. Surface flexibility prediction of M.leprae PGM. X-axis represents sequence position, while Yaxis represents surface flexibility scores.

\section{Hydrophilicity Prediction of PGM}

Parker's calculation was utilized to predict the hydrophilicity based on retention times of peptide through high-performance liquid chromatography (HPLC) on a reversed phase column [10]. Antigenicity is associated with hydrophilicity as an antigen should be accessible on the surface [24]. The hydrophilicity of PGM is represented graphically in Figure 5 where hydrophilicity is plotted based on sequence position (X-axis) against hydrophilicity prediction score (Y-axis).

By observation of Parker's hydrophilicity calculation result, it is shown that the maximum and minimum hydrophilicity predicted scores are 6.843 and -4.943 at the heptapeptide sequences $218 D E M S D D E 224$ and 100WLWIPVR106 respectively.

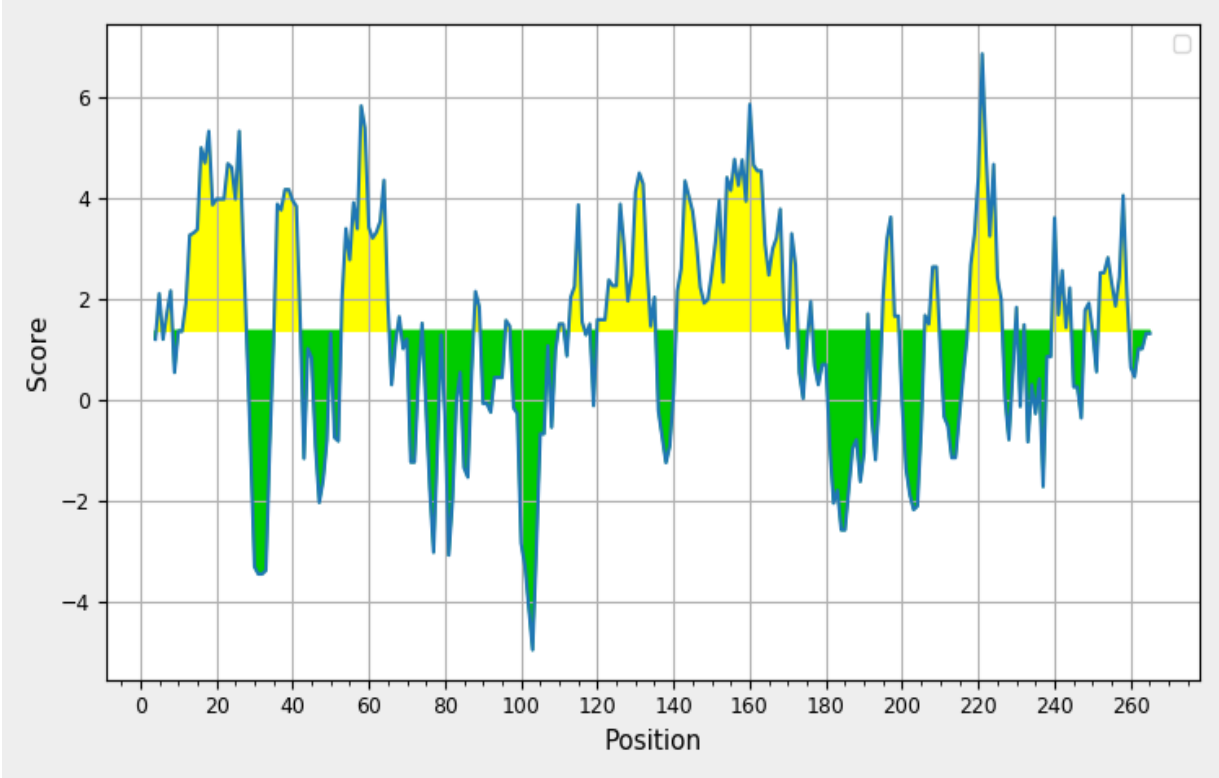

Figure 5. Parker hydrophilicity prediction for M.leprae PGM. X-axis represents the sequence position and $\mathrm{Y}$-axis represents hydrophilicity predicted score. 


\section{Prediction of CTL epitopes}

Activation of T-cells happens when the protein in the body is altered by an infectious agent and acts as an antigen. MHC molecules on the cell surface bind to the pathogenic peptide and display the infected cell for recognition by CTL, subsequently killing the cell [25]. MHC molecules exhibit high affinity to variety peptides to counteract pathogens' effort to mutate MHC epitopes [24]. Therefore, determination of T-cell epitope is a major part of computational vaccine design. This research utilized NetCTL 1.2 [26] to predict CTL epitopes from M.leprae PGM. Observed from table 2, only one CTL epitope (TADWLWIPV) has full antigenic site, while two (FSQDADPRY and YTDIGGGPL) have partial antigenic sites. These common residues inferred from both $\mathrm{B}$-cell and $\mathrm{T}$-cell epitopes prediction are considered as potential vaccine candidates.

Table 2. Identified CTL epitopes of M.leprae PGM and predicted antigenic residues (in bold). Predicted MHC binding affinity in nM units and rescale binding affinity is normalized binding affinity by first percentile score.

\begin{tabular}{ccccccc}
\hline $\begin{array}{c}\text { Residue } \\
\text { Number }\end{array}$ & $\begin{array}{c}\text { Peptide } \\
\text { Sequence }\end{array}$ & $\begin{array}{c}\text { Predicted MHC } \\
\text { Binding Affinity }\end{array}$ & $\begin{array}{c}\text { Rescale Binding } \\
\text { Affinity }\end{array}$ & $\begin{array}{c}\text { C-terminal } \\
\text { Cleavage Affinity }\end{array}$ & $\begin{array}{c}\text { Transport } \\
\text { Affinity }\end{array}$ & $\begin{array}{c}\text { Prediction } \\
\text { Score }\end{array}$ \\
\hline 26 & NTATLILLR & 0.1862 & 0.7907 & 0.5587 & 1.4870 & 0.9488 \\
\hline 94 & ALDTADWLW & 0.2477 & 1.0515 & 0.7839 & 0.9200 & 1.2151 \\
\hline 97 & TADWLWIPV & 0.1557 & 0.6609 & 0.8126 & 0.0310 & 0.7844 \\
\hline 156 & FSQDADPRY & 0.4082 & 1.7332 & 0.8713 & 2.6710 & 1.9974 \\
\hline 164 & YTDIGGGPL & 0.3579 & 1.5197 & 0.9575 & 0.8690 & 1.7067 \\
\hline 172 & LTECLADVV & 0.2219 & 0.9422 & 0.0788 & 0.0080 & 0.9545 \\
\hline 178 & DVVTRFLPY & 0.1347 & 0.5719 & 0.9570 & 2.9790 & 0.8644 \\
\hline 187 & FTDVIVPDL & 0.2693 & 1.1436 & 0.8973 & 0.6100 & 1.3087 \\
\hline 220 & MSDDEVVGL & 0.2220 & 0.9428 & 0.9618 & 0.6990 & 1.1220 \\
\hline
\end{tabular}

\section{Prediction of Structure-Based Epitopes for M.Ieprae PGM}

ElliPro is a web-based tool with the ability to correlate antigenicity characteristics, structure flexibility, and solvent accessibility of protein to predict conformational epitopes [28]. The conformational epitopes are scored based on the percentage of atoms that protrude over the molecular bulk and considered liable for antibodies to bind [28]. Seen from table 3, top three of the predicted conformational epitopes score above 0.6. The 3D structures visualization is also presented in figure 6 .

Table 3. Predicted conformational epitopes of M.leprae PGM.

\begin{tabular}{llc}
\hline No. & Residues & Number of Residues \\
\hline & A:N5, A:T6, A:A7, A:A39, A:V42, A:R43, A:E46, A:A49, A:E50, A:H51, A:N52, & Score \\
A:L53, A:L54, A:P55, A:D56, A:H71, A:L72, A:D75, A:T76, A:A77, A:D78, & 49 \\
A:W79, A:W81, A:I82, A:P83, A:T167, A:D168, A:V171, A:P172, A:D173, & \\
A:R175, A:T176, A:G177, A:R178, A:T179, A:D218, A:L219, A:D220, & \\
A:A221, A:D222, A:L223, A:R224, A:P225, A:V226, A:V227, A:P228, \\
A:G229, A:G230, A:L233 \\
A:E16, A:D18, A:A21, A:R22, A:N23, A:L24, A:F25, A:V29, A:G32, A:L33, \\
A:T34, A:D35, A:K36, A:R38, A:A97, A:G100, A:L101, A:D102, A:K103, \\
A:A104, A:V105, A:T106, A:K107, A:A108, A:R109, A:Y110, A:G111, \\
A:E112, A:E113, A:R114, A:F115, A:M116, A:A117, A:W118, A:R120, \\
A:S121, A:Y122, A:D123, A:T124, A:P125, A:P126, A:P127, A:P128, A:I129, \\
A:E130, A:K131, A:G132, A:S133, A:E134, A:F135, A:S136, A:Q137, \\
A:D138, A:A139, A:D140, A:P141, A:T144, A:D145, A:I146, A:G147, A:G148, \\
A:G149, A:P150, A:L151, A:T152, A:M199, A:S200, A:D201, A:D202, A:E203 \\
A:N208, A:P210, A:D234, A:P235, A:E236, A:A237, A:A238, A:A239, \\
A:A240, A:V241, A:I242, A:S243, A:Q244
\end{tabular}




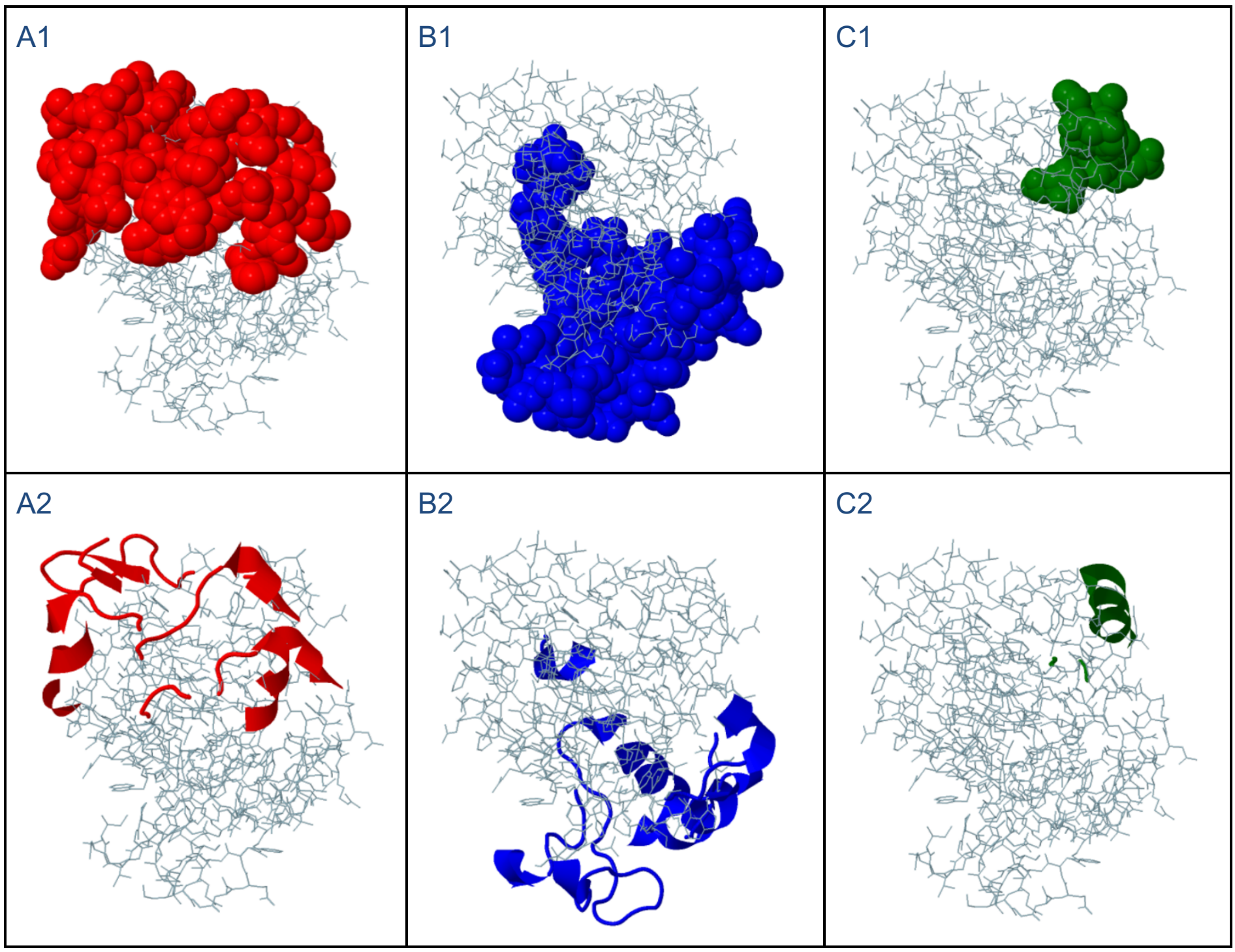

Figure 6. 3D structure of the predicted conformational epitopes of M.leprae PGM in two styles (style 1 and 2 ). Score of A is 0.685 ; score of $B$ is 0.682 ; and score of $C$ is 0.663 .

\section{Molecular Docking Analyzation of M.leprae PGM with HLA-A2}

Molecular docking analyses were performed with all three of the predicted CTL epitopes for M.leprae PGM and MHC class I HLA-A2. The binding affinities of the selected CTL based on attractive van der Waals (vdW) energy range from -16.26 to -10.29 ; repulsive vdW range from 5.81 to 3.34 ; atomic contact energy range from 7.75 to 2.56 ; hydrogen bonding range from -1.13 to 0 ; and overall global energy range from -3.31 to $-0.96 \mathrm{kcal} / \mathrm{mol}$ (Table 4). Post docking analyses were executed by using PyMol, Yasara, and UCSF Chimera and the result is the effective binding affinities of the peptide with HLA-A2, which are visualized and can be observed in figure 7 .

FSQDADPRY-MHC 1 HLA-A2 is predicted to have a stable complex because of 5 in total of hydrogen bonds with the interactions range from 2.7-3.4 $\AA$. On the other hand, YTDIGGGPL-MHC 1 HLA-A2 and TADWLWIPV-MHC 1 HLA-A2 complexes only have one and two hydrogen bonds respectively. These suggest that the two might have unstable complexes. 
Table 4. Statistical detail of M.leprae PGM-MHC class I HLA-A2 binding interactions. ACE: atomic contact energy. HB: hydrogen bonding energy

\begin{tabular}{|c|c|c|c|c|c|c|c|c|}
\hline \multirow{3}{*}{ Peptide } & \multirow{3}{*}{$\begin{array}{c}\text { Global } \\
\text { Energy } \\
\text { (kcal/mol) }\end{array}$} & \multirow{3}{*}{$\begin{array}{l}\text { Attractive } \\
\text { vdW energy }\end{array}$} & \multirow{3}{*}{$\begin{array}{l}\text { Repulsive } \\
\text { vdW }\end{array}$} & \multirow{3}{*}{ ACE } & \multirow{3}{*}{ HB } & \multicolumn{3}{|c|}{ H. Bond Interaction } \\
\hline & & & & & & \multicolumn{2}{|c|}{ Complex Pair } & \multirow{2}{*}{$\begin{array}{c}\text { Bond } \\
\text { Distance }(\AA)\end{array}$} \\
\hline & & & & & & Peptide & MHC & \\
\hline \multirow[t]{5}{*}{ FSQDADPRY } & \multirow[t]{5}{*}{-16.86} & \multirow[t]{5}{*}{-16.26} & \multirow[t]{5}{*}{5.81} & \multirow[t]{5}{*}{7.75} & \multirow[t]{5}{*}{-1.13} & SER2 OG & ASP29 OD1 & 3.438 \\
\hline & & & & & & Ser2 OG & Ser4 OG & 3.136 \\
\hline & & & & & & ARG 80 & Lys58 N & 2.841 \\
\hline & & & & & & SER2 N & ASP29 OD1 & 2.816 \\
\hline & & & & & & PHE1 N & ASP29 OD1 & 2.736 \\
\hline YTDIGGGPL & -22.28 & -24.02 & 20.61 & -0.70 & -2.55 & ASP3 OD1 & LYS6 NZ & 2.482 \\
\hline \multirow{2}{*}{ TADWLWIPV } & \multirow{2}{*}{-9.27} & \multirow{2}{*}{-10.29} & \multirow{2}{*}{3.34} & \multirow{2}{*}{2.56} & \multirow{2}{*}{0} & THR1 OG1 & GLU212 OE1 & 3.508 \\
\hline & & & & & & PRO8 O & ARG3 NH1 & 1.675 \\
\hline
\end{tabular}

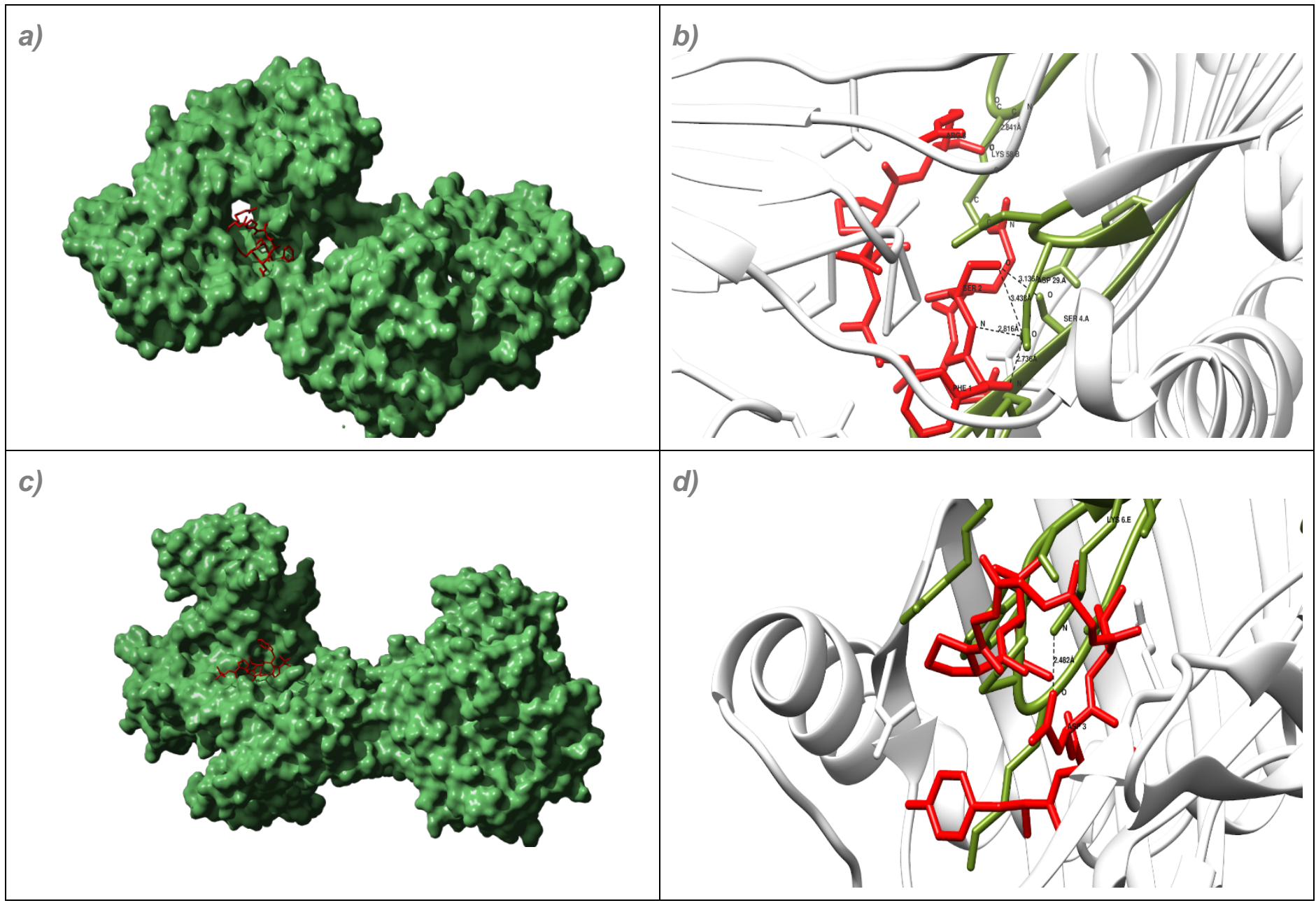




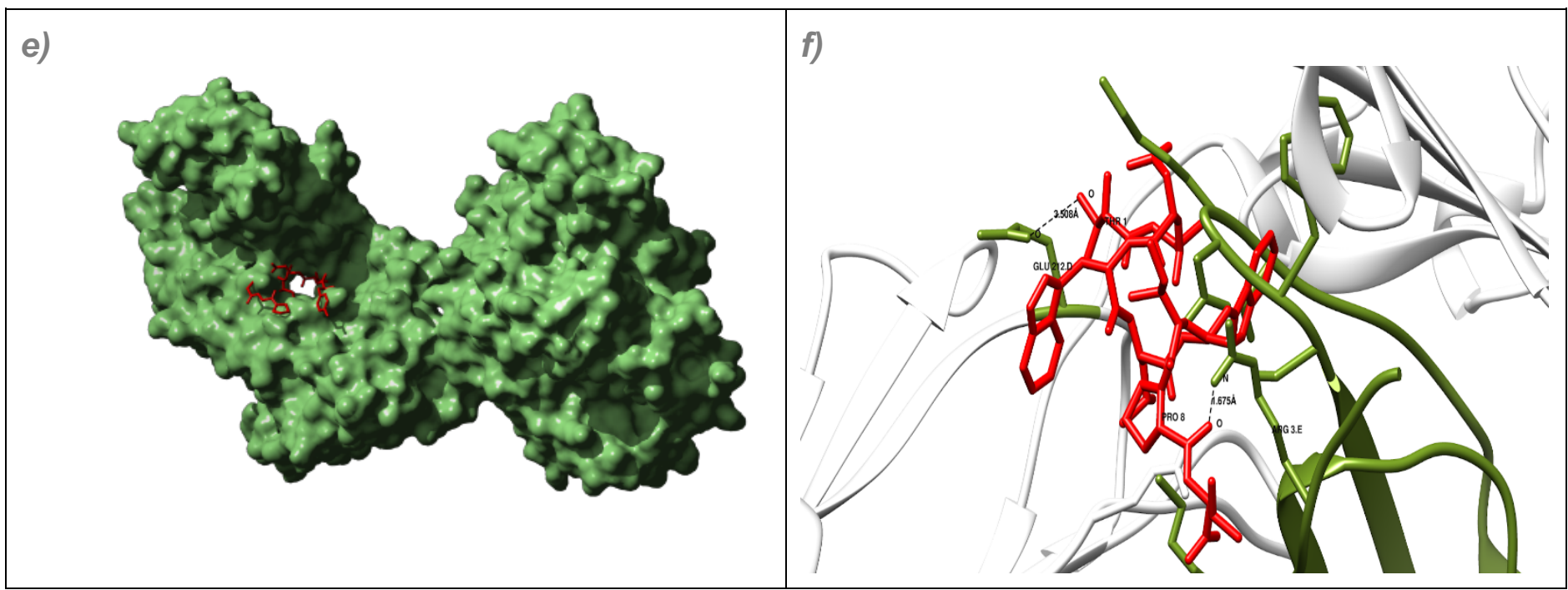

Figure 7. Binding interactions of peptide and MHC class I HLA-A2 complexes. From the top is a) hydrophobic map and b) hydrogen bond of FSQDADPRY-MHC 1 HLA-A2; c) hydrophobic map and d) hydrogen bond of YTDIGGGPL-MHC 1 HLA-A2; and e) hydrophobic map and f) hydrogen bond of TADWLWIPV-MHC 1 HLA-A2 complexes, respectively.

With new cases of leprosy increasing in India, Brazil, and Indonesia, a solution is needed. The ability to remain undetected and its asymptomatic character in the early stage, make a vaccine much more vital to eradicate the disease. In-silico methods have proven to reduce the time and resources needed to screen vaccine candidates for in-vitro experiments in vaccine design. This research predicts antigenic epitopes from M.leprae PGM, an important catalyzation that is involved in glycolysis which if inhibited will stop ATP production and impede the metabolic pathway [7]. Therefore, a vaccine that acts as a peptide inhibitor is of great interest, especially considering the rapid action and having no toxic metabolites to human health. The research also includes molecular docking as an effort to analyze the binding affinities of candidate peptides to MHC-1 protein. Previous studies have validated peptide-MHC I complex binding affinity and the utilization of computational approaches, such as Tahir et al., [24] who identify potential epitope against DENV-NS3 protein, and Mirza et al. [29], who utilized the method against Zika virus. Gupta et al., [30] has also utilized a computational method to identify vaccine or drug targets by screening the whole bacteria genome.

In this study, three peptides are predicted to show strong interaction with the MHC I HLA-A2 protein based on the global energy scores. The antigenic sites observed from the linear epitopes, which is also in the CTL epitopes prediction, are present in the selected peptides. And the antigenicity increases the chance of being a promising target for vaccine. Surface accessibility, flexibility, and hydrophilicity are also predicted to validate further with IEDB. The three predicted peptides were modeled with PEPFOLD3, followed by docking to the MHC class I HLA-A2 with PatchDock. The top models were refined by using FireDock to have stable protein structures. The resulting complexes were visualized by using PyMol, Yasara, and UCSF Chimera 1.15 to analyze the docked complexes' bonding interactions.

The three selected peptides show promise due to their antigenicity, but only one (FSQDADPRY) shows a possibility of having a stable docked complex with MHC protein HLA-A2. However, the predicted epitope does not show interactions with the binding residues of MHC I HLA A-2 (THR4, ARG97, ASP30 and HIS70) [24]. Therefore, the current study needs to be researched further to be of help in developing effective peptide vaccines in in-vitro experiment.

\section{Conclusions}

The aim is to identify potential peptides as vaccine targets to inhibit M.leprae-PGM protein. To achieve this, selected epitopes from PGM GPM1 were modeled and docked against MHC class I to analyze their interactions and binding affinities. The method was concluded with one antigenic CTL epitope that had possibility as vaccine target candidates. Further research should be conducted to validate the in-silico study 


\section{Conflicts of Interest}

The authors declare that there is no conflict of interest regarding the publication of this paper.

\section{Acknowledgment}

In this valuable chance, the researchers intended to express their gratitude and appreciation to a lot of people who have provided motivation, advice, and support. Special appreciation goes to the Institute of Research and Community Empowerment (LPPM) of Indonesia International Institute for Life Science (i3L). This paper would not have been possible without their help.

\section{References}

[1] Wibawa, T., \& Satoto, T. B. (2016). Magnitude of Neglected Tropical Diseases in Indonesia at Postmillennium Development Goals Era. Journal of tropical medicine, 2016, 5716785. https://doi.org/10.1155/2016/5716785

[2] A. Tiwari, S. Dandel, R. Djupuri, L. Mieras, J.H. Richardus, Population-wide administration of single dose rifampicin for leprosy prevention in isolated communities: A three year follow-up feasibility study in Indonesia, BMC Infect. Dis. 18 (2018) 1-8. doi:10.1186/s12879-018-3233-3.

[3] Coppola M, van den Eeden SJF, Robbins N, et al. Vaccines for Leprosy and Tuberculosis: Opportunities for Shared Research, Development, and Application. Front Immunol. 2018;9:308. Published 2018 Feb 26. doi:10.3389/fimmu.2018.00308

[4] SAGE Working Group. Report on BCG vaccine use for protection against mycobacterial infections including tuberculosis, leprosy, and other nontuberculous mycobacteria (NTM) infections. WHO. Published 2017 Sep 22. Available

https://www.who.int/immunization/sage/meetings/2017/october/1_BCG_report_revised_version_online.pdf

[5] Duthie, M. S., Gillis, T. P., \& Reed, S. G. (2011). Advances and hurdles on the way toward a leprosy vaccine. Human vaccines, $7(11), 1172-1183$. https://doi.org/10.4161/hv.7.11.16848

[6] Jaiswal AK, Tiwari S, Jamal SB, et al. Reverse vaccinology and subtractive genomics approaches for identifying common therapeutics against Mycobacterium leprae and Mycobacterium lepromatosis. J Venom Anim Toxins Incl Trop Dis. 2021;27:e20200027. Published 2021 Apr 9. doi:10.1590/1678-9199-JVATITD-2020-0027

[7] van der Oost J, Martijn A. Huynen, Corné H. Verhees, Molecular characterization of phosphoglycerate mutase in archaea, FEMS Microbiology Letters, Volume 212, Issue 1, 2002, Pages 111-120, https://doi.org/10.1111/j.1574-6968.2002.tb11253.x

[8] Emini EA, Hughes JV, Perlow DS, Boger J. Induction of hepatitis A virus-neutralizing antibody by a virus-specific synthetic peptide. J Virol. 1985;55(3):836-839. doi:10.1128/JVI.55.3.836-839.1985

[9] Karplus PA, Schulz GE. Prediction of Chain Flexibility in Proteins - A tool for the Selection of Peptide Antigens. Naturwissenschaften 1985; 72:212-3.

[10] Parker JM, Guo D, Hodges RS. New hydrophilicity scale derived from high-performance liquid chromatography peptide retention data: correlation of predicted surface residues with antigenicity and X-ray-derived accessible sites. Biochemistry. 1986;25(19):5425-5432.

[11] Kolaskar AS, Tongaonkar PC. A semi-empirical method for prediction of antigenic determinants on protein antigens. FEBS Lett. 1990;276(1-2):172-174. doi:10.1016/0014-5793(90)80535-q

[12] Ponomarenko JV, Bui H, Li W, Fusseder N, Bourne PE, Sette A, Peters B. 2008. ElliPro: a new structure-based tool for the prediction of antibody epitopes. BMC Bioinformatics 9:514.

[13] Lamiable A, Thévenet $P$, Rey J, Vavrusa M, Derreumaux P, Tufféry P. PEP-FOLD3: faster de novo structure prediction for linear peptides in solution and in complex. Nucleic Acids Res. 2016 Jul 8;44(W1):W449-54.

[14] Shen Y, Maupetit J, Derreumaux P, Tufféry P. Improved PEP-FOLD approach for peptide and miniprotein structure prediction J. Chem. Theor. Comput. 2014; 10:4745-4758

[15] Thévenet P, Shen Y, Maupetit J, Guyon F, Derreumaux P, Tufféry P. PEP-FOLD: an updated de novo structure prediction server for both linear and disulfide bonded cyclic peptides. Nucleic Acids Res. 2012. 40, W288-293.

[16] Larsen MV, Lundegaard C, Lamberth K, Buus S, Lund O, Nielsen M. Large-scale validation of methods for cytotoxic T-lymphocyte epitope prediction. BMC Bioinformatics. 2007; 8:424.

[17] Duhovny D, Nussinov R, Wolfson HJ. Efficient Unbound Docking of Rigid Molecules. In Gusfield et al., Ed. Proceedings of the 2'nd Workshop on Algorithms in Bioinformatics(WABI) Rome, Italy, Lecture Notes in Computer Science 2452, pp. 185-200, Springer Verlag, 2002

[18] Schneidman-Duhovny D, Inbar Y, Nussinov R, Wolfson HJ. PatchDock and SymmDock: servers for rigid and symmetric docking. Nucl. Acids. Res. 33: W363-367, 2005

[19] N. Andrusier, R. Nussinov and H. J. Wolfson. FireDock: Fast Interaction Refinement in Molecular Docking. Proteins (2007), 69(1):139-159.

[20] E. Mashiach, D. Schneidman-Duhovny, N. Andrusier, R. Nussinov, H. J. Wolfson. FireDock: a web server for fast interaction refinement in molecular docking. Nucleic Acids Res. (2008), 36(Web Server issue):W229-32.

[21] The PyMOL Molecular Graphics System, Version 2.0 Schrödinger, LLC.

[22] Bioinformatics 30, 2981-2982. Molecular graphics created with YASARA (www.yasara.org). 
[23] Pettersen, E.F.; Goddard, T.D.; Huang, C.C.; Couch, G.S.; Greenblatt, D.M.; Meng, E.C.; Ferrin, T.E., UCSF Chimera-a visualization system for exploratory research and analysis. Journal of computational chemistry, 2004, 25, (13), 1605-1612.

[24] Tahir RA, Wu H, Rizwan MA, Jaffar TH, Saleem S, \& Sehgal SA. Immunoinformatics and molecular docking studies reveal potential Epitope based Peptide Vaccine against DENV-NS3 Protein. Journal of Theoretical Biology. 2018. doi:10.1016/j.jtbi.2018.10.005

[25] Fishman JM, Wiles K, Wood KJ. The Acquired Immune System Response to Biomaterials, Including Both Naturally Occurring and Synthetic Biomaterials. Host Response to Biomaterials. Academic Press 2015; $151-$ 187. doi.org/10.1016/B978-0-12-800196-7.00008-6

[26] Janeway CA Jr, Travers P, Walport M, et al. Immunobiology: The Immune System in Health and Disease. 5th edition. New York: Garland Science; 2001. The major histocompatibility complex and its functions. Available from: https://www.ncbi.nlm.nih.gov/books/NBK27156/.

[27] Larsen, M.V.; Lundegaard, C.; Lamberth, K.; Buus, S.; Lund, O.; Nielsen, M., Large-scale validation of methods for cytotoxic T-lymphocyte epitope prediction. BMC bioinformatics, 2007, 8, 424

[28] Ponomarenko J, Bui HH, Li W, et al. ElliPro: a new structure-based tool for the prediction of antibody epitopes. BMC Bioinformatics. 2008;9:514. Published 2008 Dec 2. doi:10.1186/1471-2105-9-514

[29] Mirza MU, Rafique S, Ali A, Munir M, Ikram N, Manan A, Salo-Ahen OM, Idrees M. Towards peptide vaccines against Zika virus: Immunoinformatics combined with molecular dynamics simulations to predict antigenic epitopes of Zika viral proteins. Scientific reports, 2016, 6, 37313.

[30] Gupta, E., Gupta, S.R.R. \& Niraj, R.R.K. Identification of Drug and Vaccine Target in Mycobacterium leprae: A Reverse Vaccinology Approach. Int J Pept Res Ther 26, 1313-1326 (2020). https://doi.org/10.1007/s10989019-09936-x 